\title{
Collaborative Problem Solving
}

\section{I Background}

As already mentioned in the first chapter, an important strand of CI research addresses problem solving that involves direct interaction in smaller groups or teams. A number of these studies have identified a general ability of a group to perform on a wide variety of tasks, indicating that groups, in the same way as individuals, also have a general intelligence (Malone, 2018: 32; Woolley et al., 2010, 2015). Although the individual intelligence of the group members is relevant, both the correlation between the average and maximum intelligence of the group members and the group's CI is only moderately strong. Factors such as group satisfaction and group motivation are not significantly correlated, adding more uncertainty to what group processes are most important (Malone, 2018: 33-34, 4I-42).

Woolley et al. (2015) suggest that CI involved in collaborative problem solving in smaller groups is influenced by a complex interaction of both bottom-up (e.g., interpersonal skills) and top-down processes (group structures, norms, and routines) during problem-solving. Malone points to the importance of the three following factors: (I) individual intelligence, (2) working well with others, (3) cognitive diversity (Malone, 2018: 4I-42). At present, studies both cover offline and online settings, but we still know little about the factors that influence on CI processes or this collaborative problem-solving ability. However, current CI studies suggest that at least four group factors are important for collaborative problem solving:

- Working well with others

- Cognitive diversity

- Equal participation

- Joint coordination 
These four factors will be analyzed by reviewing current CI studies directed towards small group collaboration, and other relevant publications by wellknown CI researchers. In all these areas, there is also extensive research from outside of the CI research context, but it is out of the scope of this chapter to address these.

Instead, case studies from virtual teams in online innovation contests are included because they represent authentic problem solving in a highly relevant CI context (IdeaConnection). In some contests, individuals are handpicked for the teams. Sixteen experts are divided into three or four teams and these teams are then given I 2 weeks to compete against each other in solving the problem. In this contest format, seekers only receive a few high-quality good solutions. This reduces review time compared with innovation challenges that include hundreds of submitted ideas, many of low quality. In the teams, participant motivation is also often high, since the chance of winning is greater. In addition, a facilitator supports the team in the problem-solving process (IdeaConnection, 20I9a, 20I9b, 20I9e). The data from these teams provide insight into how advanced collaborative problem solving in the online setting can emerge in a natural setting.

\subsection{Working Well with Others}

Several CI studies find that that the ability of the group members to work well with others is important. This factor is typically measured as social perceptiveness by tests like "Reading the Mind in the Eyes" (BaronCohen, Wheelwright, Hill, Raste, \& Plumb, 200I) that requires that the test person judge others' emotions from looking only at pictures of their eyes. Group members vary significantly in how well they perform this task, and the average social perceptiveness score of the group members has been found to have a significant positive influence on the group's CI. It indicates that social perceptiveness is a measure of a person's social intelligence (Malone, 2018: 33-34, 4I-42).

Interestingly, one study shows that the social perceptiveness was equally good at predicting collective intelligence in both face-to-face groups and online groups (Engel, Woolley, Jing, Chabris, \& Malone, 2014). The result is surprising because the online groups could not observe each other, but only type text messages. It suggests that social perceptiveness is predictive of much broader interpersonal skills (Woolley et al., 2015). For example, the skill that enables you to read emotions in people's faces may perhaps make you sensitive to guessing what other people are feeling, even when they are only experienced through written text in an online 
setting (Malone, 2018: 37). Alternatively, the social perceptiveness may have something to do with qualities in the group interaction, such as active listening skills.

There is still significant uncertainty around the relational dimension in CI. The reason is that the c-factor is not predicted by several other factors that typically predict well-functioning groups, such as group satisfaction, social cohesiveness, and psychological safety concerning interpersonal risks (Woolley et al., 2015). In addition, few CI studies examine group interaction data and lack ecological validity, since they are experimental studies. For instance, there may be important differences between time-limited ad hoc groups and permanent groups. It is also likely that both specific individual relational skills and specific qualities in the group interaction are important. Top solvers in innovation teams in online innovation contests are assumed to inform our understanding of this relational dimension in collaborative problem solving and the types of group interaction that are important in CI.

\subsection{Being in a Symmetrical Group Relationship}

First, the perception of the group relationship appears to be important. Some solvers explicitly state that the team relationship evolves through the collaboration in itself. They highlight the unusually symmetrical collaborative relationship with less group hierarchy in the online setting. One solver states:

In the academic world, like everywhere else there is also ego and self-promotion. But when you work in a team environment, it is very important to keep an open mind and to be critical but also respectful. It is a very delicate balance to maintain. Some collaborations just do not work because people don't want to change their minds. But when you have a successful team, it is like magic, because when people are interacting with each other to learn and teach each other, a great trust develops. These are teams of equals and there is less hierarchy.

According to the solver, this "team of equals" emerges when people learn and teach each other during the group work. Interestingly, the solver claims that this process of learning and teaching together creates "great trust," which allows groups members to be both critical and respectful (see Section 8.4). Other solvers underline how learning and group relationships are intertwined. One solver says, "The fact that there is always something to learn from other team members makes me look forward to meetings every time." The joy of learning together is an important part of the motivation. Another solver highlights the multidisciplinary team effort, 
"One of the challenges I recently worked on was completely out of my field, and the interest there is getting to know people from a different discipline and to see how they interact, how they work together and what their strengths are. That's really what I enjoy, I think, most of all.”

If valuable peer learning is present, this has a positive influence on the group's ability to work well with each other in these high-performing teams. However, there are also challenges in the group dynamics. One solver explains:

The discussions are fun, usually anyway. Of course, when you meet people online that you've never met before you will get along well with some, but others are going to annoy you a little if they hog the conversations. Some people don't know when to be quiet and listen. So that's when the skills of a facilitator really come to the fore.

This solver's statement suggests that listening skills are important. If the group struggles, a facilitator will help support the process.

\subsubsection{Interest in Meeting People Who Are Different}

Second, several solvers report that they enjoy being together with the others in the team. One solver says, "A lot of the time you meet interesting people with interesting occupations just by working in a team." This statement suggests a curiosity and interest in meeting new people that might be of special importance in this type of collaboration between people who do not previously know each other. Another solver describes it as both fun and interesting to learn about others' different cultural backgrounds, "It is nice to see how various team members with different backgrounds come up a solution to particular challenge in a novel way. We usually have fun times during meetings and exchange cultural backgrounds to get know each other well." Another solver also underlines the joy of being in a good team, "The different personalities, the experiences and the conversations. Then there is the camaraderie, which is incredible." Here, the term "camaraderie" is used, perhaps indicating a close relationship that is not friendship, but closer to just being engaged in group work. A third solver even misses the team afterwards: "And you know, when the whole thing was over I missed talking to the team. We got to know each other more than just as co-workers. It was nice. I really did miss the whole process when it was over." When the solver misses the team, it indicates positive feelings towards work, but also that many do not keep contact after the work is over. 
Furthermore, some underline the excitement of getting to know people with a different background and expertise; one solver even describes it as "love":

I love it and I love meeting all the different people. I am working with engineers, chemists, physicists and mathematicians and they all come at these problems with different philosophies and with a different academic background. They are giving different slices of their own expertise. I think it is extremely synergistic and it keeps me on my toes. I have to be sharp so I can debate with different experts on an equal level.

The solver describes how motivating it is to meet people with different academic backgrounds. Some solvers establish long-term relations with other solvers, both by expanding their social and professional networks:

Yes, and there's also ' $c$ ' and that is I get to meet lots of interesting people. For me it is meeting those people and putting them in my network so when I am faced with a new challenge I call them up. I launch companies with my networks. I'm always looking for good people, those personalities that really mesh well with me and vice versa and have skills that complement my own.

This solver is explicit about the goal of finding people who have relevant expertise and can be part of a future network. In authentic settings, some solvers will want to work well with others because they have a strategic interest in establishing long-term business relations.

If we compare all these statements, the excitement of meeting different people stands out. One solver even describes team members as typical extroverts, "I also found people to be very, very open, and very accommodating. I think people who do open innovation by default are quite open minded." Solvers are motivated by getting to know new people. The social dimension of "working well with others" points to both specific personality characteristics and group characteristics, such as being in a symmetrical group relationship.

\subsection{Cognitive Diversity}

Another important factor in CI research orientated towards collaborative problem solving is cognitive diversity (Woolley et al., 2015). Here, the emphasis is not primarily on the different types of information other persons can bring to the table, but it is about the different thinking styles and perspectives that individuals use when they solve a task. Cognitive diversity is especially valuable in creative problem solving, which relies on 
new perspectives. Today, there is more interest in examining how groups of diverse problem solvers can outperform groups of more homogeneous high-ability performers (Page, 2017). Several areas provide strong evidence of diversity bonuses. One example is in academic research, where the percentage of teamwork has increased steadily in nearly all areas. For example, in social sciences, 20 percent of the papers were coauthored in I960, and in 2000, this numbers had increased to 50 percent of the papers. Teams also perform better in science with coauthored papers earning more citations (Wuchty, Jones, \& Uzzi, 2007).

The value of cognitive diversity will also depend on variation in perspectives (Page, 2017). For example, one top solver states that different backgrounds in the team can help produce more ideas:

Overall, it was a great experience working on the team. I do like to work with other people, and the one challenge that I've worked on so far, required a lot of theoretical work that needed expertise in many fields so I don't think one person could've solved it successfully. The challenge needed various backgrounds [...] In the group you hear many different points of view which gives you more ideas and helps you to think of things from different perspectives.

When hearing "many different points of view" this can stimulate idea development. Another solver even actively seeks to increase cognitive diversity by wanting to work with individuals that think differently:

They have to be able to work with me. There's a wonderful saying a couple of guys in my network and I came up with - if we think the same about everything, if we think identically, one of us is redundant. I want people who challenge me and look at problems in completely different ways than I do and look for solutions in their realms of expertise and experience that are dramatically different from mine. People that come into my networks are very diverse and extremely different.

The statement illustrates that some persons appear to be more aware of being with others who "look at problems in completely different ways than I do." The emphasis on perspectives that are "dramatically different from mine" indicates an attempt to maximize cognitive diversity. However, some CI studies indicate that the most collectively intelligent groups are those that are moderately diverse in cognitive styles. If the cognitive styles in the group are too diverse, group members communicate less effectively with one another (Aggarwal, Woolley, Chabris, \& Malone, 2015; Woolley et al., 2015).

An effective team must be able to share idea and acknowledge dissent. They also need to feel safe, respected, and validated. These conditions are 
important in groups that are able to combine and improve ideas (Page, 2017). For example, one solver highlights how the team is able to refine solution:

Overall, my view of IdeaConnection is that it is a great innovation because when somebody thinks alone they might think their idea is great but it may not be. When you are a part of a team if your idea is not quite there you can correct it as others are contributing. And when everyone is giving their opinions you get a very good refined solution in the end. It also connects people from different walks of life. I've been able to work with people from India, Canada and the Netherlands at the same time. I think the IdeaConnection concept is great.

In good teams, ideas will be continuously corrected or modified through contributions from every group member. Another solver explains how cognitive diversity enhances individual learning: "I also learned a lot about other disciplines, other ways of thinking through a problem, styles of writing, styles of solving complex issues, and how to integrate our different perspectives into a coherent whole." The individual learning is not only about acquiring knowledge of other disciplines, but about observing how others solve problems.

Furthermore, research studies have found a connection between cognitive diversity and identity diversity (Page, 20 I7: I7I). Identity diversity or social category diversity refers to distinctions that are made between people who are like me (in-group) and people who are not like me (out-group), typically involving factors like, gender, nationality, ethnicity, or age, but also "non-visible" characteristics like sexual orientation. While most people intuitively acknowledge the value of functional background diversity, it is less obvious that socially diverse groups can have the same effects. First, identity diversity can be a source of cognitive diversity because individuals with more different backgrounds will bring in more variation in experiences that likely also increase the cognitive diversity. However, this is not a hard-and-fast rule; two persons with different identity do not automatically bring different cognitive perspectives to the table, nor does two persons from the same identity group automatically bring identical cognitive perspectives. There will always be some degree of both cognitive and identity diversity in a group. Still, it is important to assume that identity differences among group members like gender and race can promote cognitive diversity because one will then also be more open to approaching the same problem in different ways.

A second benefit of identity diversity is simply that persons who observe differences on the surface tend to assume that there are more cognitive differences in the group. This prompts them to seek out this information. 
For example, including women in a group with only men is one way of stimulating cognitive diversity in groups. Difficult decisions often benefit from diverse representation. The mere presence of identity outsiders can change the behavior of the identity majority and potentially enhance group performance. Even if a person who is "different" does not bring in more cognitive differences, the mere presence has been shown to change the behavior of the group's members (Phillips, 2017).

One important reason is that people work harder in identity-diverse environments compared with homogeneous environments in their attempts to benefit from cognitive diversity. In this context, the groups are more positive and accepting towards alternative viewpoints. Studies show that persons who interact with individuals they perceive as different expect that it will require an effort to reach agreement (Phillips, 20I7).

Studies have also found that racial diversity can promote critical thinking. For example, one study compared homogeneous groups of six white jurors with mixed groups comprising four white and two black jurors. The white jurors in mixed groups raised more novel case facts, identified more missing evidence, and were more accurate in the discussion compared with whites in homogeneous groups. One possible explanation is that individuals in heterogeneous groups expect more disagreement to be present in the group, and therefore examine the case more thoroughly. The perceived presence of identity diversity decreases conformity to socially similar others in a group and makes it easier for everyone to speak up with more confidence (Phillips, 2017: 233-235; Sommers, 2006).

Just being exposed to diversity can change the way you think. In contrast, it may be more difficult to utilize cognitive diversity if there are no perceived triggers from identity differences. A group of individuals with similar identity traits will easily create stronger expectations of consensus. Group members care more about maintaining relationships and harmony if they are together with identity-similar others (Phillips 20I7). When hiring people, we have a tendency to falsely believe that people who share our identity are smarter and more capable. For example, studies show that biases range from 5 to 25 percent for salary offers. Small biases can also accumulate to form large biases, such as when a person needs to pass ten biased hurdles to reach the top of a company. These biased decisions can also be unintended and unconscious and may trigger nepotism (Page, 2017: 2I3-2I 5). A striking example is a study which found that, when homogenous groups were outperformed by diverse groups, the homogenous groups still reported greater confidence and effectiveness (Phillips, 2017: 233-236; Phillips, Liljenquist, \& Neale, 2009). People will filter both what they are saying 
and how they hear information, depending on who they are talking to and who is sharing that information. Individuals are often cautious in their support for diversity because they fear potential downsides. Studies show significant resistance against identity diversity because it is likely to result in more conflicts, disagreement, and questioning of one's own perspectives and opinions. These challenges require hard work that group members do not necessarily want. There is also a risk that outsiders are not respected or do not speak up because they do not feel welcome in the group. More identity diversity can cause discomfort, a lack of trust and mutual respect, communication barriers, and greater perceived interpersonal conflict. It can undermine the commitment to a group's goal, and it will not always be possible to observe immediate benefits. If some group members are more respected than others, group norms must also be reorganized to ensure that everyone's ideas are presented (Phillips, 20I7).

On the other hand, if the group members become too similar to each other in cognitive style, they will lack the variety of perspectives and skills needed to perform well (Aggarwal et al., 201 5; Malone, 20 I 8: 36; Woolley et al., 2015). If groups become too similar, they risk becoming echo chambers, and reinforce each other's existing opinions. Members may become more interested in getting along than critically evaluating each other's ideas. For CI, it is crucial to bring in a sufficient diversity of perspectives (Woolley et al., 2015).

Organizations typically attempt to utilize cognitive diversity by combining it with functional background diversity (Page, 20I7). For example, several of the solvers highlight the value of multidisciplinary diversity:

So on a previous challenge on the prediction of the fate of organic chemicals in soil, the seeker was looking for a model and we had a statistician on the scene and without a statistician we would've been dead in the water. But the statistician didn't know any chemistry and didn't know how these things degraded. So separately we would've been useless but together we were a good team.

This solver claims the problem could not have been solved without the different academic backgrounds in the team. Another solver explains the value of including geographical diversity in an online setting:

For instance, we had people from South America, Canada, the U.S., and so having people from different climates provided insight into different crops, times of year, soil types, just real on the ground practical information. If you had just a number of folks in a university in one particular city it might be difficult to get all those types of insights. 
In this case, informational diversity is utilized because individuals from different parts of the world can easily participate in the problem-solving process. One solver also emphasizes the importance of complementary rather than overlapping expertise:

I think the difference is that the challenges at IdeaConnection tend to have a group of people who probably wouldn't be working together in the sense that they have complementary rather than overlapping expertise. So you're now putting people talking together who think differently and also have a different primary dataset on which they're basing what they're talking about. So you have a much more widely read community in lots of ways at that point.

All these examples illustrate how the online setting makes it easy to design teams that would not normally be working together. These groups build on both multidisciplinary and multicultural diversity, which can potentially utilize cognitive diversity in new ways.

\subsection{Equal Participation}

Equal participation is another important factor for CI in collaborative problem solving. Several studies shows that equality of communication and work contribution among group members is important, both in faceto-face and online groups. When one or two people dominate the conversation, the group is on average less intelligent compared with groups that have a more evenly distributed participation and conversational turntaking (Engel et al., 2014; Malone, 2018: 35-36; Woolley et al., 2010, $2015)$. One argument is the fair sharing of the workload. Another is that equal participation aims to utilize diverse member skills by involving everyone. However, group dynamics will often hinder this openness because group members think their opinion is irrelevant or they may fear disapproval from others (Landemore, 2013; Sunstein, 2006). In a classical experiment, Stasser and Titus (1985) showed that groups discussing a political problem are often surprisingly bad at using all the information they possess. Sometimes, group discussions even lead to worse decisions.

In the experiment, three written profiles of fictional president candidates of the student government at a university were created. The profiles contained information about the candidates' policies on issues of interest to students like dorm visitation hours and local drinking ordinances. They deliberately constructed three profiles so that one of the candidates was clearly more desirable than the other two. In the first version of the experiment, each student received complete profiles of all three candidates, 
and, not surprisingly, 67, percent of the students chose the best candidate. When students were then divided into small groups of four persons, the support for the best candidate increased to 85 percent (Nielsen, 201 I: 69-70).

However, in the second version of the experiment, the researchers altered the profiles so that each student only received partial information about the three candidates. Some of the positive information about the best candidate was removed, and in addition some of the negative information about the undesirable candidates. Every group member would therefore receive information that suggested that one of the undesirable candidates was better than the best candidate. As a result, 6I percent then individually chose the undesirable candidate. Afterwards, students were divided into small groups of four with all information available about all three candidates. Still, the support for the undesirable candidate increased from 6I percent to 75 percent and the support for the best candidate decreased from 25 to 20 percent. It showed that groups were not sharing information in an efficient way, and they performed worse than the average member in the group. In a 1989 follow-up of the same experiment, the researchers found that the main weakness was that the group spent most of their time discussing information they had in common and did not use time exploring all available information. When several members had negative information about the best candidate, this was perceived as more important than the positive information held by only a single member. Groupthink ignores information from others even when most students think it is important to pool information from everyone. Another follow-up study found that asymmetrical relationships amplify the negative influence on group decisions. Unique information held by low-status members was much more likely to be ignored (Nielsen, 20 I I: 70-72).

Although few CI studies provide any detailed characteristics of equal participation in collaborative problem solving, the design of cognitive diversity will obviously be relevant. Groups who emphasize the value of a diversity of perspectives will strive to involve all group members (Phillips, 2017: 237-242). If we look at the online innovation teams, several top solvers also mention the value of equal participation. One solver states:

We all had different contributions which is what made it fun and stimulating. I looked forward to our discussions together. We did phone conferences and the team was so respectful of each other's backgrounds. We really worked hard to incorporate all our backgrounds into the final product. And I felt the theoretical portion which I contributed was honored as much as the technical content. The way we worked together was a wonderful experience and an example of how to truly collaborate and listen to each other. 
Here, the solver emphasizes that all contribute, but differently. The team is "respectful of each other's backgrounds," honoring each other's work and tries to "incorporate all our backgrounds into the final product." The ability to "listen to each other" is an important part of this process. Here, equal participation is about cognitive diversity by respecting and listening to others in the group. Because these teams are multidisciplinary and each individual has unique competence, it might be more obvious to let everyone voice their opinion. Another solver also describes how equal participation involves having different roles and tasks in the group work:

In the first way, as it went along we started to naturally fall into different roles on the team and I think that helped. Initially as four team members, we were trying to split things equally into four, and then, I think, we would get kind of frustrated if two people held up their end of that bargain and two people were lackadaisical about it or maybe procrastinating a little bit. Whereas, eventually we ended up where we naturally fell into distinct roles.

This solver explains that the teams initially split the work into four identical parts, but this still led to some persons doing more work than others did. If some individuals are free riding, this can threaten the group work. With the support of the facilitator, the team managed to reorganize the work.

Because $\mathrm{I} 2$ weeks is a short period to solve a scientific problem, building trust in the virtual team is crucial. Solvers will often need to work with tasks that do not fully fall under their own expertise. Some will need weeks to understand the basic terminology, which can potentially create difficulties in sustaining the work because members work part time. Others may quit before the project is finished. When the solvers work in teams, a typical source of conflict will be members who do not do their part of the task. Nor may there be enough time to solve all relevant issues in the team meetings (Arnold, 2019a; Hossain, 2018). In order to design equal participation, it is necessary to understand "what people want to achieve" and how they can contribute to the group. However, the mix of expertise can also be challenging, as one solver states:

So it got better as I got to understand where the other people were in their careers and what their background was in terms of whom should work on which part of the project, who should be working together, and who should be editing things in terms of making sure that everything is coherent. It got easier as it went on. There was a lot of standing off at the beginning.

In this example, the work improved when the group found out who should work on which part of the project. It underlines the importance 
of explicitly discussing tasks to ensure equal participation. Regarding this issue, the solvers highlight the importance of the facilitators:

$\mathrm{He}$ [the facilitator] submits the solutions, which is good, and writes them up. He wants to get contributions from everybody. It's like, say you're the foreman of a jury, and everyone has to vote, the foreman wants to get everyone's opinion. It's the same thing here; a facilitator makes sure everybody contributes. And he'll delegate the work and so on.

The prominent role of a facilitator illustrates that teams will often benefit from a skilled person who can help organize the work in an effective way. It is likely that these groups to a much larger degree would have failed, like in the Stasser Titus experiment, if they were left to self-organize. Equal participation is without doubt an important design principle in collaborative problem solving that builds on CI, as numerous empirical studies and case stories show. It is not a principle that groups will automatically organize themselves around.

\subsection{Joint Coordination}

The fourth important CI factor in collaborative problem solving is joint coordination. Current studies find that the amount of spoken communication is important, both in face-to-face groups and written dialogue in online groups (Engel et al., 2014; Woolley et al., 2015). It is possible that more communication stimulates a stronger shared practice and more joint coordination of the problem-solving process. Previous sections highlighted both cognitive diversity and equal participation. However, there needs to be a balance between maintaining divergence and establishing a common understanding or shared goal. If the group share a body of knowledge and strategies, it is more likely they will resolve disagreements (Page, 2017: I73; Phillips, 2017). However, we need to better understand how this joint coordination can be achieved in collaborative problem solving. The data from the innovation teams suggest that at least four different coordination mechanisms are relevant:

I. Establishing a shared understanding of the problem

2. Planning the process

3. Staying focused on the shared goals

4. Ensuring the conversational flow

These coordination processes are heavily influenced by a facilitator who supports the process (Arnold, 2019b; IdeaConnection, 2019d). Without this person, it is likely that this type of coordination will become more difficult. 


\subsection{Establishing a Shared Understanding of the Problem}

First, it is important to establish a shared understanding of the problem. In the first phase, teams may ask for clarification of the problem to make sure that they avoid misunderstandings. One solver explains how the group approached the seeker, asking for more information:

Yes, and I thoroughly believe in spending some time on analyzing the problem because the solution doesn't come out of thin air. It comes out of looking deeply into the problem, the context, what has been done in parallel industries or what has been done before. The more information you have, the easier it is to hone in on the right solution. I find the meeting with the seeker a very important part of the solution, because we really need to listen to them and try to learn from their experience. As a team we have experience, but not experience of these direct problems. Therefore, the more we can learn from the seeker the easier it is for us to come up with a solution that will match their needs.

Here, the solver underlines the importance of listening to the seeker to really understand the problem. New solutions will usually build on versions of solutions that already exist. A solver even looks at this clarification process as a way of stimulating the development of new ideas.

\subsubsection{Planning the Process}

Second, it is necessary to outline a plan for the teamwork. In the innovation teams, the facilitator will be important in ensuring this is done. The facilitator proposes an outline that help the group members divide the tasks. One solver explains this process:

Right away the facilitator came up with an outline that she felt would answer the challenge and we took parts of that outline that were most appropriate to our backgrounds. This outline was so helpful and I just adapted my theory to my parts of the outline as did the other two solvers. We divided that outline in about five minutes. It was painless.

By providing a structure and time plan, the joint coordination work becomes much more efficient.

\subsubsection{Staying Focused on Shared Goals}

Third, it is important to stay focused on the shared goals. In the innovation teams, the solvers underline how the facilitator helped the team stay focused: 
She was more of a moderator than a Facilitator because sometimes the conversation would veer off into what I would call unproductive areas. Having the Facilitator there cut the conversation short and kept us focused on trying to get things done in the time we had allocated. I think that part was very critical for me because my time was limited.

The facilitator kept the group on track by stopping "unproductive" conversations, which is important because time is limited. Another solver illustrates how the group is highly focused on finding a solution and nothing else:

Well, it's much different than being part of groups in a company. I think everybody is much more focused on the solution rather than focusing on building their career or getting to lunch or other distractions you have in a small group in a company. So the focus is much more laser-like and directed. It is very pleasant to work with such focused people and experts in all of their fields. Everybody I've worked with is an expert in their field and it's good to get a glimpse of what they're doing at the cutting edges of their fields.

Although the group is diverse, with group members from many different fields, the group is still very goal orientated. When all efforts only need to be directed towards the cognitive effort of solving the problem, performance can increase. In addition, a solver states that the facilitator helps summarize the work, "The facilitator helps a lot. First of all, the facilitator looks at the problem and focuses us on it. And he communicates all our hopes and solutions into a coherent summary. And we look at the summary and see a trend in the thinking. The facilitator helps us to come up with the solution." By having a person summarizing the collective work, this can potentially help the group synthesize their efforts.

\subsubsection{Ensuring the Conversational Flow}

Fourth, it is important ensure the conversational flow. One solver says:

A fundamental part of any team is the facilitator. They are responsible for half of the team's success by keeping the pace and rhythm and solving any relationship problems if they come up. The other half is due to the team's technical background and having the time and the will to do the job.

Here, the facilitator is vital in keeping up the pace and sustaining the discussions. Part of the challenge is that individuals work differently, as one solver states, "Then, the other challenging part of it, I think, was just learning to work with people that you didn't know previously. We all seemed to have different styles of working and different styles of 
communicating. But by the end, I felt good about the way we handled it and the way we ended up coming together." This group struggled because they wanted to work and communicate in different ways. However, they managed to come together and agree on a shared group strategy. Another solver also emphasizes that the facilitator is important when such conflicts are present, "You need somebody to have that authority in the team, especially when one person says this way is right, and someone else says the other way is right." It is important that the facilitator help settle disagreements in an impartial way. This reduces the likelihood of new conflicts and sustains the conversational flow in a better way. 